Check for updates

Cite this: RSC Adv., 2017, 7, 56117

Received 8th August 2017

Accepted 27th November 2017

DOI: $10.1039 / c 7 r a 08775 f$

rsc.li/rsc-advances

\title{
Front-face fluorescence spectroscopy of tryptophan and fluorescein using laser induced fluorescence and excitation emission matrix fluorescence
}

\begin{abstract}
Stéphane Mounier, (D) *ab Roland Redon, ${ }^{a}$ Gustavo Nicolodellib and Debora Milorib
Herein we study the responses of two standard molecules, tryptophan and fluorescein, over a large concentration range in boric acid via two front-face fluorescence spectroscopies: excitation emission matrix fluorescence and laser-induced fluorescence. The responses in relation to the tryptophan content were measured and were found to depend on the measurement technique. For fluorescein, no response was found for the excitation emission matrix, and an inverse relationship with the solid content was observed for the measured laser-induced fluorescence. Aiming to enhance the very weak fluorescence response of fluorescein, a novel protocol using a gel interface was established. These methods represent a future premise to investigate molecules with low fluorescence efficiency on surfaces using a logarithmic response.
\end{abstract}

\section{Introduction}

Front-face fluorescence spectroscopy (FFFS) is a powerful method to detect and determine fluorescent molecules in solid matrices without contact and sample preparation. It allows rapid and efficient characterization and quantification in pharmaceutical or alimentary domains. In environmental or agricultural applications, this technique could be a powerful tool for pollutant or nutrient quantification. However, FFFS was developed essentially in the agronomic domain using the fluorescence of the chlorophyll family to determine plant health, ${ }^{\mathbf{1}}$ for lake sediment surveys ${ }^{2}$ and for red wine monitoring. ${ }^{3}$ Very few applications were based on other samples such as soils, ${ }^{4}$ sewage or compost. ${ }^{5}$ The application of FFFS in the environmental domain is under development because the samples present complex mixtures of several mineral matrices as well as complex and simple organic molecules. Hence, before using FFFS as a routine technique for heterogeneous surfaces in environmental systems, it is necessary to study several key parameters including diffusive effects, color or absorbance, the chemical and physical states of surfaces, and theoretical models. These parameters cannot be treated simultaneously, and simpler systems must be studied first to progressively increase the complexity. This work addresses the relationship between the FFFS responses and the molecule content in a solid. Compacted boric acid or simple powder mixture

${ }^{a}$ Laboratoire PROTEE, Université de Toulon, CS 60584, 83041 Toulon Cedex 9, France. E-mail: stephane.mounier@univ-tln.fr; Fax: +33 49414 2157; Tel: +33 494142829 ${ }^{b}$ EMBRAPA Instrumentação, Rua XV de Novembro, 1452-Centro, São Carlos, SP, Brazil matrices were studied. Tryptophan, a well-known and wellstudied molecule, and fluorescein, a molecule used as a fluorescence standard for solutions, were chosen to investigate two FFFS techniques: laser induced fluorescence (LIF) and emission excitation matrix fluorescence (EEM). Tryptophan is a fluorescent amino acid and is present in all proteins and consequently, in every biological material. Its fluorescence properties depend on its chemical environment and its structural location. ${ }^{6}$ Whatever its state, solid or in solution, the more efficient excitation wavelength domain is 250 to $280 \mathrm{~nm}$, and the emission wavelength domain ranges from 310 to $380 \mathrm{~nm},{ }^{6-8}$ depending on the chemical conditions. Pure tryptophan is a white yellowish solid. Fluorescein fluorescence in solution occurs in the excitation wavelength domain of 440 to $500 \mathrm{~nm}$ and its emission occurs from 510 to $516 \mathrm{~nm}$ without an emission wavelength shift except under extreme conditions.9,10 Solid fluorescein is a dark red powder. No previous report was found on the solid-state fluorescence of fluorescein. In this work, tryptophan and fluorescein were studied in the solid state over a wide range of content, from $0.0002 \%$ to $7.618 \%$ and from $0.002 \%$ to $77.92 \%$ for fluorescein and tryptophan, respectively. Fluorescence emission was measured by FFFS with LIF and EEM to establish the relationship between the molecule content and fluorescence response in a solid boric acid matrix.

\section{Materials and methods}

\section{Sample manufacturing}

Boric acid powder (BA, Fisher Chemical) was used to prepare solid pellets with different tryptophan (TRP) and fluorescein 
(FLU) content. An initial solid mixture was created by finely grinding a boric acid mass with a TRP or a FLU mass, giving the highest solid content of C0-TRP and C0-FLU, respectively (Table 1). After this, known masses of C0-TRP and C0-FLU were added to another mass of BA to prepare the next set of samples C1-TRP and C1-FLU, respectively, and so on, to obtain a nearlogarithmic decrease of TRP and FLU content in the sample set (Table 1). The zero content pellet was obtained using only the BA solid. For each solid mixture, one part of the powder was pressed at 8 tons per $\mathrm{cm}^{2}$ to obtain two solid pellets. Eventually, measurements were performed on the pellets and powders for 13 and 14 different content samples ranging from $2 \times 10^{-3}$ to $78 \%$ for TRP and $2 \times 10^{-4}$ to $7.6 \%$ for FLU, respectively. The content in percentage of mass for TRP and FLU is denoted as \% TRP and \% FLU, respectively. All solids were conserved in a dark, dry, enclosed plastic bag to avoid contamination and degradation. All amounts and references are given in Table 1.

\section{EEM acquisition}

EEM measurements were taken on a Perkin Elmer LS50B spectrofluorimeter using a solid holder module while measuring the pellet under quartz glass. The excitation range was from 200 to $400 \mathrm{~nm}$ varying every $10 \mathrm{~nm}$ with the excitation slit fixed at $5 \mathrm{~nm}$. The corresponding emission spectra were acquired from 200 to $600 \mathrm{~nm}$ with a scan speed of $250 \mathrm{~nm} \mathrm{~min} \mathrm{~m}^{-1}$ and a slit of $5 \mathrm{~nm}$. No emission filter was used. The response integration time and the photomultiplicator tension were both set as automatic. The 10 by $10 \mathrm{~nm}$ extractions of the excitation emission matrices were obtained via the 3D export tool from the Perkin Elmer program FLWinLab.

\section{PARAFAC analysis}

To determine the component's source contribution to the EEMs, the CP/PARAFAC algorithm was used. First all EEMs were cleaned from the scattering signals: the Rayleigh by cutting the scattering band $(20 \mathrm{~nm})$ and the Raman from first and second diffraction orders by applying the Zeep procedure. ${ }^{11}$

Table 1 Tryptophan (TRP) and fluorescein (FLU) content in \% of mass and code

\begin{tabular}{llll}
\hline Code & $\begin{array}{l}\text { FLU (\% } \\
\text { of mass) }\end{array}$ & Code & $\begin{array}{l}\text { TRP }(\% \\
\text { of mass) }\end{array}$ \\
\hline C0-FLU & 7.618 & C0-TRP & 17.32 \\
C1-FLU & 3.766 & C1-TRP & 8.518 \\
C2-FLU & 2.505 & C2-TRP & 5.545 \\
C3-FLU & 1.364 & C3-TRP & 3.043 \\
C4-FLU & 0.7949 & C4-TRP & 1.306 \\
C5-FLU & 0.5929 & C5-TRP & 0.4205 \\
C6-FLU & 0.3790 & C6-TRP & 0.2726 \\
C7-FLU & 0.2845 & C7-TRP & 0.1812 \\
C8-FLU & 0.2044 & C8-TRP & 0.0923 \\
C9-FLU & 0.1017 & C-TRP & - \\
CA-FLU & 0.0158 & CA-TR & 77.92 \\
CB-FLU & 0.0045 & CB-TRP & 39.46 \\
CC-FLU & 0.0008 & CC-TRP & 0.0100 \\
CD-FLU & 0.0002 & CD-TRP & 0.0021
\end{tabular}

Decomposition was then performed from two to six components on the 28 corrected EEMs, and the higher number of components giving a CORCONDIA test over $60 \%$ was selected as the optimal component number. ${ }^{12,13}$ No outliers were present in the data and 3 components were found. Because the fluorescence gives a response depending on the apparatus, the component contribution was noted by relative contribution to the fluorescence (RCF). The RCF of a component should be related to the molecule content and is comparable from one sample to another. ${ }^{12}$ The CP/PARAFAC model components were indexed as $\mathrm{C}_{\text {TRP-FLU }}$, where $X$ is the component number and TRP-FLU indicates the name of the database.

\section{LIF measurement}

The laser-induced fluorescence was measured with a portable LIF system (lab-made equipment developed by EMBRAPA Instrumentation, Sao Carlos, Brazil) equipped with a $405 \mathrm{~nm}$ laser source. ${ }^{\mathbf{1 4}}$ An optical fiber was coupled with a solid sample holder. Each face of each pellet was measured under the following conditions: emission wavelength range from 450 to $800 \mathrm{~nm}$, integration time $1 \mathrm{~s}$, boxcar 4 , and average 5 . For each measurement, the integration of the fluorescence emission spectra was denoted as $A_{\mathrm{LIF}}^{\mathrm{X}}$ expressed in arbitrary units of fluorescence, a.u. by nm, where $\mathrm{X}$ is the studied molecule TRP or FLU.

\section{Gel interface measurements}

A gel interface was tested to enhance the fluorescence response of the solid state. A hydroxy-gel was synthesized on the same day of the measurement. Tetraethylorthosilicate (TEOS) was added to $\mathrm{HCl}$ until the $\mathrm{pH}=3$. Hydrolysis was performed under gentle stirring for $36 \mathrm{~h}$. After this, ethanol was added to the solution, and the gel was extracted by centrifugation, cleaned with ethanol and conserved in a glass container at $4{ }^{\circ} \mathrm{C}$ in the dark. Two grams of the lump gel was placed on the quartz glass of the sample holder. By applying gentle pressure with the counter piece, the gel sliced and became transparent. After removal of the counter piece, the gel remained as a layer, sticking to the quartz windows, with slight cracks. Ten milligrams of powder was then deposited on the gel surface, and the system was pressed again until the gel cracks disappeared. The gel layer width was approximately 1 to $2 \mathrm{~mm}$ following pressure application. During the stabilization time, the emission fluorescence $\left(\lambda_{\mathrm{ex}} / \lambda_{\mathrm{em}}=490 / 510 \mathrm{~nm}\right)$ was measured every minute to monitor the response evolution. This protocol was performed for the 14 pellets containing fluorescein and the boric acid pellet.

\section{Results and discussion}

\section{Direct solid EEM decomposition}

The CP/PARAFAC decomposition of the fourteen EEM measurements of the TRP and FLU pellets gives three independent components (CORCONDIA 81.71\%) presented in Fig. 1. Component $\mathrm{C} 1_{\text {TRP-FLU }}$ (Fig. 1a) is defined by a peak maximum at $\left(\lambda_{\mathrm{ex}} / \lambda_{\mathrm{em}}\right)=250 / 325 \mathrm{~nm}$ with an ellipsoidal shape. Component C2 $2_{\text {TRP-FLU }}$ (Fig. 1b) is slightly shifted compared to 


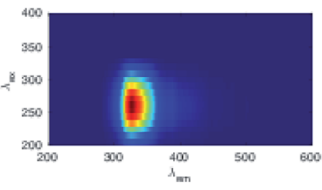

a)

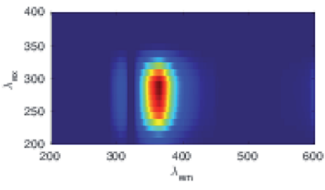

b)

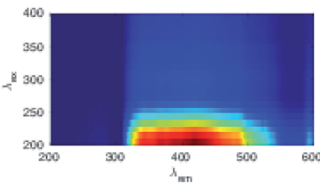

c)

f)

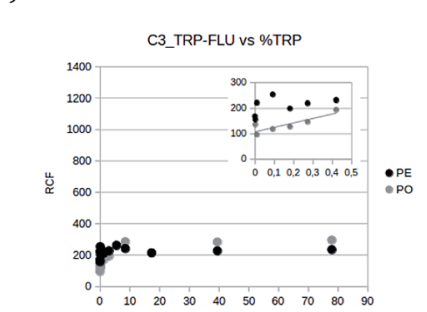

Fig. 1 CP/PARAFAC components from the whole set of pellets and powders in the TRP-FLU data set $(\mathrm{a}-\mathrm{c})$ and their RCF related to the TRP content in mass $(d-f)$. PE and PO represent the pellet and powder responses, respectively. The inset graphs focus on low TRP content ranging from 0 to $1 \%$ in mass.

C1 $1_{\text {TRP-FLU }}$ with a peak maximum at $\left(\lambda_{\text {ex }} / \lambda_{\text {em }}\right)=280 / 380 \mathrm{~nm}$ and a sharper ellipsoidal shape. These two locations are near the fluorescence of tryptophan in solution. ${ }^{6-8,15,16}$ One can note that the $\mathrm{C} 1_{\mathrm{TRP}-\mathrm{FLU}}$ excitation range is narrower than that of

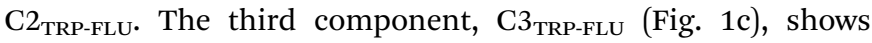
a horizontal ellipsoidal peak centered on $\left(\lambda_{\mathrm{ex}} / \lambda_{\mathrm{em}}\right)=210 / 400 \mathrm{~nm}$ with a large emission range from 320 to $500 \mathrm{~nm}$. C3 $3_{\text {TRP-FLU }}$ seems to be related neither to fluorescein nor tryptophan but to a non-specific solid surface response. ${ }^{17}$ The relationship between components $\mathrm{C} 1_{\text {TRP-FLU }}$ and $\mathrm{C} 2_{\text {TRP-FLU }}$ with the TRP content in percent (Fig. 1d and e) shows a rapid increase of the response from 0 to $1 \%$ and a saturation from $1 \%$ to $80 \%$ for the tryptophan content. The same behavior was observed for $\mathrm{C} 2_{\text {TRP-FLU }}$ with a higher RCF for the powder than for the pellet. Moreover, this difference persists at the low range of tryptophan ( 0 to $1 \%$ ), and it is obvious that a linear relationship exists only for the powder state with a good correlation coefficient for both $\mathrm{C}_{\text {TRP-FLU }}\left(r^{2}=0.93\right.$, slope $=2355 \mathrm{RCF} \%$ of $\left.\mathrm{TRP}^{-1}\right)$ and $\mathrm{C} 2_{\text {TRP-FLU }}\left(r^{2}=0.94\right.$, slope $=610 \mathrm{RCF} \%$ of $\left.\mathrm{TRP}^{-1}\right)$ as shown in the insets of Fig. 1(d) and (e), respectively. For the pellets, the RCF directly plateaus just below the saturation value (Fig. 1d and e). The only difference between the powder and the pellets is the solid surface texture, and reflection or diffusive effects could explain this difference. Concerning $\mathrm{C}_{\text {TRP-FLU }}$, which was attributed to the solid state, it presents no variation $\left(r^{2}=0.38\right.$, slope $=103$ RCF\% of TRP ${ }^{-1}$ ) (Fig. 1f), except for the low \% of TRP that could be attributed to an incomplete separation of the TRP fluorescence by CP/PARAFAC; i.e., TRP fluorescence emission occurs up to $380 \mathrm{~nm}$ and this could influence the decomposition, leading at low content to a linear relationship with the $\%$ of TRP.

\section{Direct solid LIF spectra}

LIF spectra measurements are presented in Fig. 2a-d; each individual spectrum is the average of the four measured spectra from the two pellets (two faces for two pellets of the same TRP content) and two measured spectra for the powder (duplicate). There is little difference in the spectrum shapes of the powder and pellet states for the signal of TRP (Fig. 2). The correlation between the pellet and powder responses is very strong; $r^{2}=$ 0.94 and $r^{2}=0.95$ for the TRP area and maximum intensity, respectively, whereas it is less correlated for FLU, where $r^{2}=$ 0.83 and $r^{2}=0.84$ for the area and maximum intensity, respectively. This means that for TRP, the solid state does not influence the response, whereas for FLU, it is more significant. Regardless of this, the fluorescein response presents a maximum intensity, $I_{\mathrm{LF}, \mathrm{Max}}^{\mathrm{FLU}}$, at $520 \mathrm{~nm}$ and several slight shoulders at $480 \mathrm{~nm}, 575 \mathrm{~nm}$, and $620 \mathrm{~nm}$. This emission location, $520 \mathrm{~nm}$, is slightly red shifted compared with the FLU emission in solution, which is at $510 \mathrm{~nm}$. Concerning tryptophan, the maximum intensity, $I_{\mathrm{LIF}, \mathrm{Tax}}^{\mathrm{TRP}}$, is centered at $490 \mathrm{~nm}$, and only one shoulder appears at $520 \mathrm{~nm}$. This emission peak
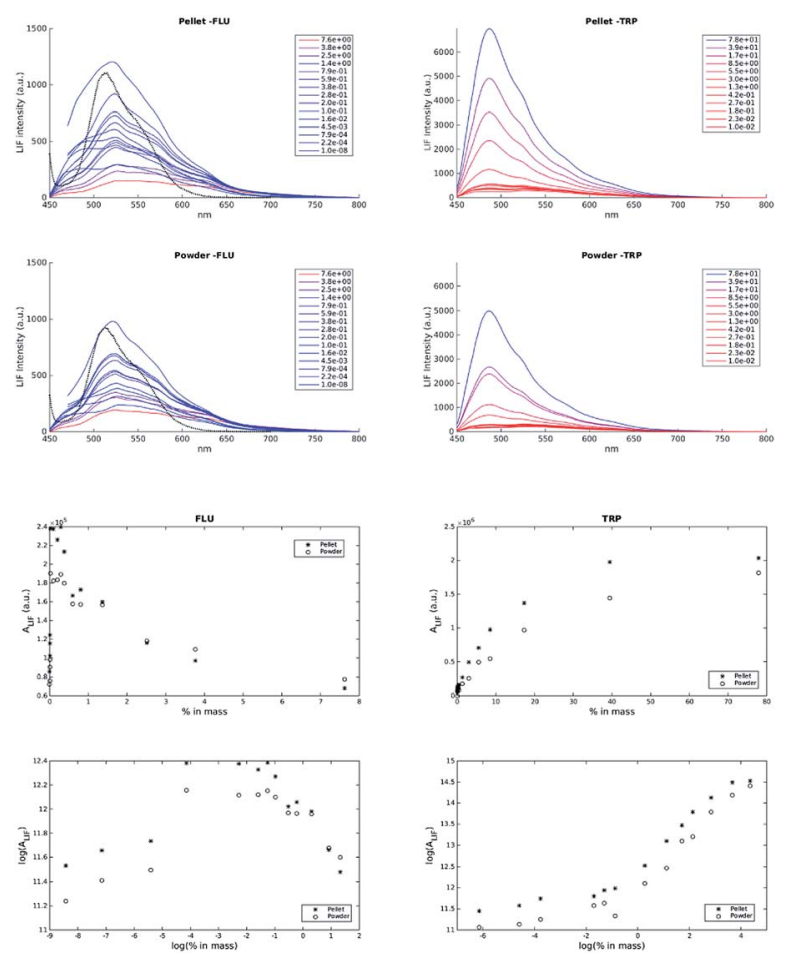

Fig. 2 Mean LIF spectra of FLU in pellet (PE-FLU) and powder (POFLU) forms and of TRP in pellet (PE-TRP) and powder (PO-TRP) forms. The dotted line spectra represent the fluorescein spectra in solution. 
position for TRP was not expected compared to the emission maximum for TRP in solution, which is between $310 \mathrm{~nm}$ and $380 \mathrm{~nm}$. The variation of the area under the curve $A_{\mathrm{LIF}}^{\mathrm{FLU}}$ and $A_{\mathrm{LIF}}^{\mathrm{TRP}}$ versus percent of chemical, and the corresponding $\log \left(A_{\mathrm{LIF}}^{\mathrm{FLU}}\right)$ and $\log \left(A_{\mathrm{LIF}}^{\mathrm{TRP}}\right)$ versus the logarithm of the chemical content, are described in Fig. 2 for both powder and pellet states. For FLU, there is little difference between the pellet and powder responses. Hence, for pellets or powders, $A_{\mathrm{LIF}}^{\mathrm{FLU}}$ decreases exponentially with increasing FLU content; this is unexpected behavior for a specific fluorescence response. This could be because the fluorescein solid state color induces a strong inner filter effect. However, because fluorescence is isotropic, some part of the fluorescence emitted by molecules located at the surface should be observed because it irradiates directly out of the solid. Another hypothesis could be drawn by understanding this phenomenon as a strong quenching effect by the neighbor of the fluorescein molecules, either the boric acid or the fluorescein itself. This hypothesis could be supported by the linear correlation $\left(A_{\mathrm{LIF}}^{\mathrm{FLU}}=50010 \times \%\right.$ of FLU +408$)$ observed at a very low content of FLU $(<0.1 \%)$, which is strong $\left(r^{2}=0.95\right)$. The tryptophan LIF response, $A_{\mathrm{LIF}}^{\mathrm{TRP}}$, shows a logarithmic trend curve with a lower response for the powder than for the pellet state in Fig. 2. This saturation phenomenon could be explained by an inner filter effect like in solution ${ }^{\mathbf{1 8}}$ but must be theoretically demonstrated for solids. However, for the low \% of TRP, the relationship is linear until $\log (\%$ of TRP $)=-2$. Below this $\%$ of TRP, $A_{\mathrm{LIF}}^{\mathrm{TRP}}$ does not vary when the blank response is reached. By using a linear regression it is possible for the tryptophan to determine a detection limit, in the solid boric acid matrix, of $0.022 \%$ tryptophan in mass.

\section{EEM with gel interface results}

The emission spectra of fluorescein extracted using a gel are shown in Fig. 3(a), for three different stabilization periods $(0,10$ and $35 \mathrm{~min}$ ) after gel contact and for the fluorescein powder C8 (0.2022\% fluorescein in mass). During the diffusion of the fluorescein in the gel, the intensity increases and there is no shift in the peak position. Fig. 3b presents the maximum fluorescence intensity $(510 \mathrm{~nm})$ normalized to the initial intensity $(t=0 \mathrm{~s})$, a few seconds after the deposition of the powder in the gel, as a function of time, except for the C3-FLU content (550 $\mathrm{nm})$, owing to the saturation of the detector at the
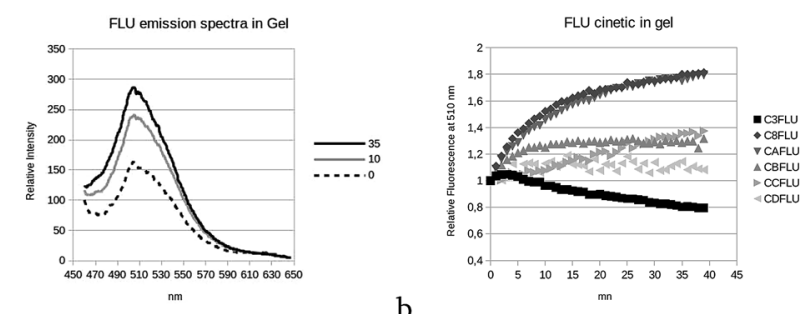

a $\mathrm{b}$

Fig. 3 Emission spectra of fluorescein in a gel layer (a) after 0, 10, and $35 \mathrm{~min}$ of stabilization. Kinetics of the fluorescence response of fluorescein with powders of different content during the diffusion into the gel at the maximum intensity of fluorescence (b). maximum wavelength. For the C3-FLU content, the intensity increases slightly and decreases regularly with time (Fig. 3b, black squares). This is due to the inner filter effect of fluorescein, which becomes highly concentrated in the gel. For lower concentrations (from C8-FLU to CD-FLU), the stabilization follows a logarithmic rule, and the stabilization time is reached more rapidly when the content is low. For the CD-FLU content (Fig. 3b, light-gray triangles), the intensity is very low, and the signal is too noisy to detect a stabilization trend. Considering this stabilization time, the FFFS-EEM fluorescence measurement was performed after $1 \mathrm{~h}$ of stabilization to ensure that there was no evolution with time that influenced the results. The system was then stabilized for $1 \mathrm{~h}$, and the FFFS-EEM measurement was taken with the same parameters described previously.

The CP/PARAFAC decomposition of all the gel-extracted fluorescein samples gives three components (CORCONDIA $88.51 \%$ ), which are presented in Fig. 4. Component $\mathrm{C} 1_{\mathrm{FLU}-\mathrm{GEL}}$ shows a large domain of excitation with a maximum at $400 \mathrm{~nm}$ and a straight emission band centered at $510 \mathrm{~nm}$. It is slightly blue shifted compared to the emission range of the fluorescein in solution. The second component, $\mathrm{C} 2_{\mathrm{FLU}-\mathrm{GEL}}$, presents an excitation maximum at $450 \mathrm{~nm}$ and the same emission as $\mathrm{C} 1_{\mathrm{FLU}-\mathrm{GEL}}$. The range of excitation for $\mathrm{C} 1_{\mathrm{FLU}-\mathrm{GEL}}$ and $\mathrm{C} 2_{\mathrm{FLU}-\mathrm{GEL}}$ of the fluorescein in the gel is not consistent with those found in the literature, $480-500 \mathrm{~nm}$. This blue shift could be explained by conformational influence due to the gel network. However, the redshift excitation position of $\mathrm{C} 2_{\mathrm{FLU}-\mathrm{GEL}}$ compared to that of $\mathrm{C} 1_{\text {FLU-GEL }}$ could be attributed to the inner filter effect for the fluorescein with high concentration. ${ }^{18}$ Indeed, it was not possible to apply an inner filter effect correction to the FFFS measurements because the surface absorbance measurement is difficult and because no theoretical model allows this correction. When the concentration of the fluorescein increases in the gel, the auto-absorption process shifts the excitation peak to higher wavelengths such as those that occur in solution. This redshift of emission wavelength, normally observed when the fluorescein is in solution, does not occur in this experiment. The C3 $3_{\text {FLU-GEL }}$ component fluorescence maximum is located at the $\left(\lambda_{\text {ex }} / \lambda_{\text {em }}\right)=200 / 400 \mathrm{~nm}$ position. It seems that this component is similar to $\mathrm{C} 3_{\text {TRP-FLU }}$ and specific to the FFFS-EEM technique and not related to the samples in this work.

The RCF of $\mathrm{C} 1_{\mathrm{FLU}-\mathrm{GEL}}, \mathrm{C} 2_{\mathrm{FLU}-\mathrm{GEL}}$ and $\mathrm{C} 3_{\mathrm{FLU}-\mathrm{GEL}}$ after $1 \mathrm{~h}$ of stabilization is presented in Fig. 5. The RCF of $\mathrm{C} 1_{\text {FLU-GEL }}$ is linear for the low content of fluorescein of $<0.02 \%$ in mass (Fig. 5, inset, $r^{2}=0.99$ ) but dramatically decreases after this
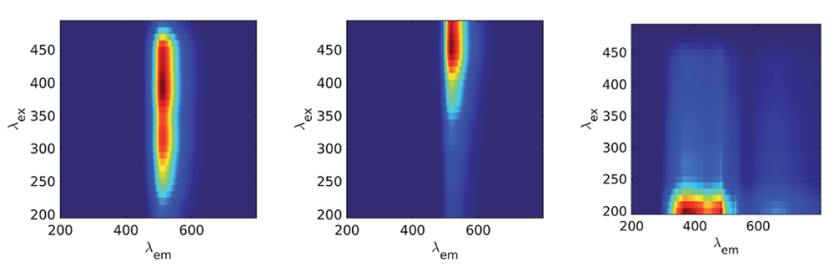

Fig. 4 CP/PARAFAC components for the EEM fluorescence measurement of fluorescein (FLU) in the presence of the gel layer. 


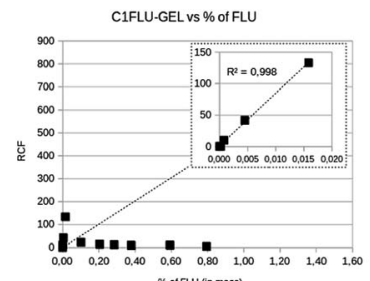

a)

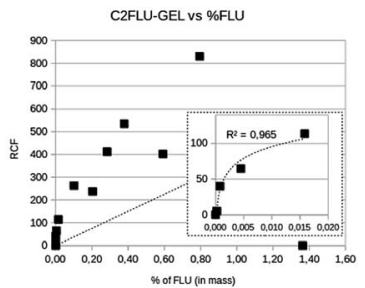

b)

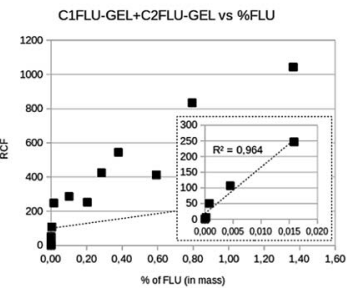

c)

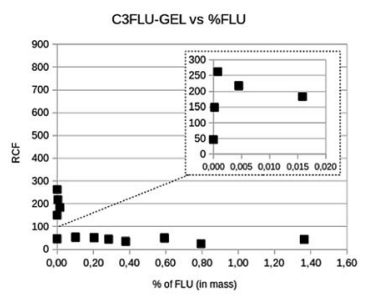

d)

Fig. 5 CP/PARAFAC relative contribution of CX FLU-GEL versus the FLU content in the solid powder.

content limit (Fig. 5a). The $\mathrm{C}_{\text {FLU-GEL }}$ component contribution increases with increasing fluorescein content. However, there is a difference of slope between the lower content and the higher content (Fig. $5 \mathrm{~b}$ ) owing to the very low contribution of $\mathrm{C} 2_{\mathrm{FLU}-\mathrm{GEL}}$ from 0 to $0.005 \%$ of fluorescein in mass. In fact, assuming that $\mathrm{C} 1_{\mathrm{FLU}-\mathrm{GEL}}$ and $\mathrm{C} 2_{\mathrm{FLU}-\mathrm{GEL}}$ are related to the two responses of fluorescein in the presence of the inner filter effect in the gel, it can be considered as the sum of contributions between $\mathrm{C} 1_{\mathrm{FLU}-\mathrm{GEL}}$ and $\mathrm{C} 2_{\mathrm{FLU}-\mathrm{GEL}}$. Hence, the relationship clearly presents two lines of correlation, one under $0.022 \%$ of fluorescein $\left(r^{2}=0.96\right.$, slope $=14825 \mathrm{RCF} \%$ of $\left.\mathrm{FLU}^{-1}\right)$ and another one over this limit, with a lower correlation coefficient and slope $\left(r^{2}=\right.$ 0.87 , slope $=630 \mathrm{RCF} \%$ of $\mathrm{FLU}^{-1}$ ) (Fig. $5 \mathrm{c}$ ). The $\mathrm{C}_{\mathrm{FLU}-\mathrm{GEL}}$ component is unrelated to the fluorescein content and presents a non-linear increase for the first point, after which it decreases regularly to reach an average contribution of 500 RCF (Fig. 5d).

\section{Conclusions}

The solid state fluorescence responses of tryptophan and fluorescein in boric acid depend on the spectroscopic technique and content. For tryptophan, the FFFS-EEM gives a response that fits well with the fluorescence spectral location of tryptophan in solution and it confirms the possibility of measuring this compound directly in solid samples. The CP/PARAFAC decomposition in a data-set of EEM with a mass content between 0 and $80 \%$ of fluorescein or tryptophan gives three components. Two are highly related to the tryptophan fluorescence and give a linear response for low content ( 0 to $1 \%$ of TRP) with a saturation of the contribution over $1 \%$. The relative contribution to the fluorescence response of the components at a low content of TRP clearly depends on the solid state: the powder gives a linear response, whereas the pellets saturated the detector, probably due to the diffusive or reflective effects. The FFFS-LIF response of tryptophan shows a linear trend with different slopes for the powder and pellet forms. However, the LIF fluorescence occurs at an unusual wavelength $\left(\lambda_{\mathrm{ex}} / \lambda_{\mathrm{em}}\right)=$
$405 / 495 \mathrm{~nm}$ for tryptophan and further investigations have to be performed to understand which physical processes are involved. For fluorescein, no response was obtained for the FFFS-EEM technique, whereas using FFFS-FIL, a decreasing spectral response was obtained in the emission wavelength range for fluorescein in solution. This behavior could be due to a very strong inner filter effect attributed to the fluorescein color (red-brown), and this hypothesis should be tested.

By interposing a silicon gel between the quartz glass and solid powder, it was possible to obtain a fluorescein response using the FFFS-EEM technique. The CP/PARAFAC components show a fluorescence peak at the same emission position as fluorescein in solution, but with a $90 \mathrm{~nm}$ blue shift for the excitation wavelength. A piecewise linear relationship was observed with a steep slope from 0 to $0.02 \%$ of fluorescein in mass and a less steep slope over this limit. No saturation of the signal was observed with the gel interface during this experiment. For all FFFS-EEM measurements, a component located at $\left(\lambda_{\mathrm{ex}} / \lambda_{\mathrm{em}}\right)=200 / 400 \mathrm{~nm}$ was found after CP/PARAFAC separation, which can be attributed to the solid-state response or to the apparatus response. For fluorescein, it was possible to measure some fluorescence by FFFS-EEM using a silicon gel layer, but further investigations should be performed to obtain quantitative results. However, even without preparing a solid surface measurement, this gel method could be developed to easily and rapidly measure heterogeneous surfaces like soils or coated materials that normally exhibit weak or no fluorescence.

\section{Conflicts of interest}

There are no conflicts to declare.

\section{Acknowledgements}

This work was supported by Professor Visitante Especial, Ciença sem Fronteira on Fluorescence Response on Heterogeneous Surfaces [processo No 313576/2013-0]. This work was supported by the French government [2015-UTLN-CRCT] and the FUI project BAC-TRACK (AAP-21/DOS0039705/00). We thank Dr Elaine Cristina Paris from EMBRAPA Instrumentation of Sao Carlos, Brazil, who provided the gels for this work.

\section{References}

1 D. M. B. P. Milori, M. Raynaud, P. R. Villas-Boas, A. L. Venancio, S. Mounier, R. B. Bassanezi and R. Redon, Comput. Electron. Agr., 2013, 95, 11-18.

2 D. Airado-Rodríguez, T. Galeano-Díaz, I. Durán-Merás and J. P. Wold, J. Agric. Food Chem., 2009, 57, 1711-1720.

3 M. Woszczyk, A. Bechtel, R. Gratzer, M. J. Kotarba, M. Kokocinski, J. Fiebig and R. Cieslinski, Org. Geochem., 2011, 42, 1025-1038.

4 M. Muller, D. M. B. P. Milori, S. Déléris, J. P. Steyer and Y. Dudal, Waste Manag., 2011, 31, 1916-1923.

5 R. Albrecht, E. Verrecchia and H. R. Pfeifer, Talanta, 2015, 134, 453-459.

6 J. T. Vivian and P. R. Callis, Biophys. J., 2001, 80, 2093-2109. 
7 M. Tabak, G. Sartor and P. Cavatorta, J. Lumin., 1989, 43, 355-361.

8 C. P. Pan, P. L. Muiño, M. D. Barkley and P. R. Callis, J. Phys. Chem. B, 2011, 115, 3245-3253.

9 R. Sjöback, J. Nygren, M. Kubista, R. Sjback, J. Nygren and M. Kubista, Spectrochim. Acta, Part A, 1995, 51, L7-L21.

10 X. He, G. Guoquan, Z. Hui and L. Hu-lin, Microchem. J., 1997, 331, 327-331.

11 R. G. Zepp, W. M. Sheldon and M. A. Moran, Mar. Chem., 2004, 89, 15-36.

12 R. Bro and H. A. L. Kiers, J. Chemom., 2003, 17, 274-286.
13 S. Mounier, H. Zhao, C. Garnier and R. Redon, Biogeochemistry, 2011, 106, 107-116.

14 C. H. Santos, G. Nicolodelli, R. A. Romano, A. M. Tadini, P. R. Villas-Boas, C. R. Montes, S. Mounier and D. M. B. P. Milori, J. Braz. Chem. Soc., 2015, 26, 1136-1142.

15 M. Tedetti, P. Joffre and M. Goutx, Sens. Actuators, B, 2013, 182, 416-423.

16 D. M. Reynolds, Water Res., 2003, 37, 3055-3060.

17 S. Mounier, G. Nicolodelli, R. Redon and D. Milori, Spectrochim. Acta, Part A, 2017, 177, 79-85.

18 X. Luciani, S. Mounier, R. Redon and A. Bois, Chemom. Intell. Lab. Syst., 2009, 96, 227-238. 